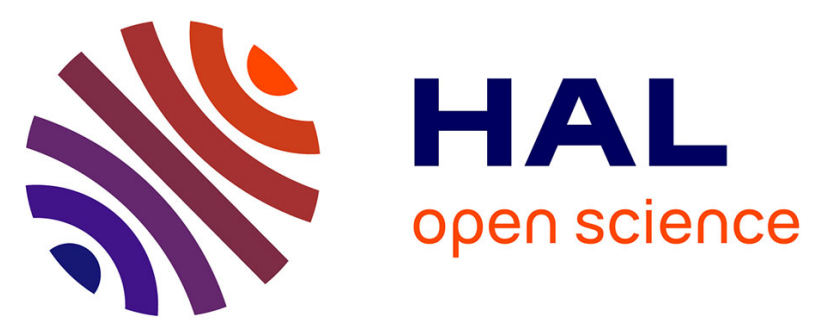

\title{
Time-Difference-of-Arrival Estimation Based on Cross Recurrence Plots, with Application to Underwater Acoustic Signals
}

\author{
Olivier Le Bot, Cedric Gervaise, Jerome I. Mars
}

\section{To cite this version:}

Olivier Le Bot, Cedric Gervaise, Jerome I. Mars. Time-Difference-of-Arrival Estimation Based on Cross Recurrence Plots, with Application to Underwater Acoustic Signals. Charles L. Webber, Jr.; Cornel Ioana; Norbert Marwan. Recurrence Plots and Their Quantifications: Expanding Horizons, 180, Springer, pp 265-288, 2016, Springer Proceedings in Physics, 978-3-319-29921-1. 10.1007/978-3319-29922-8_14. hal-01343668

\section{HAL Id: hal-01343668 \\ https://hal.science/hal-01343668}

Submitted on 8 Jul 2016

HAL is a multi-disciplinary open access archive for the deposit and dissemination of scientific research documents, whether they are published or not. The documents may come from teaching and research institutions in France or abroad, or from public or private research centers.
L'archive ouverte pluridisciplinaire HAL, est destinée au dépôt et à la diffusion de documents scientifiques de niveau recherche, publiés ou non, émanant des établissements d'enseignement et de recherche français ou étrangers, des laboratoires publics ou privés. 


\title{
Time-difference-of-arrival estimation based on cross recurrence plots, with application to underwater acoustic signals
}

Olivier Le Bot, Cédric Gervaise and Jérôme I. Mars

\begin{abstract}
The estimation of the time difference of arrival (TDOA) consists of the determination of the travel-time of a wavefront between two spatially separated receivers, and it is the first step of processing systems dedicated to the identification, localization and tracking of radiating sources. This article presents a TDOA estimator based on cross recurrence plots and on recurrence quantification analysis. Six recurrence quantification analyses measures are considered for this purpose, including two new ones that we propose in this article. Simulated signals are used to study the influence of the parameters of the cross recurrence plot, such as the embedding dimension, the similarity function, and the recurrence threshold, on the reliability and effectiveness of the estimator. Finally, the proposed method is validated on real underwater acoustic data, for which the cross recurrence plot estimates correctly $77.6 \%$ of the TDOAs, whereas the classical cross-correlation estimates correctly only $70.2 \%$ of the TDOAs.
\end{abstract}

Olivier Le Bot

Univ. Grenoble Alpes, GIPSA-Lab, Grenoble F-38000,

CNRS, GIPSA-Lab, Grenoble F-38000

e-mail: lebotol@gmail.com

Cédric Gervaise

Chaire Chorus, Foundation of Grenoble INP, 46 Avenue Félix Viallet, 38031 Grenoble cedex 1

e-mail: cedric.gervaise @gipsa-lab.grenoble-inp.fr)

Jérôme I. Mars

Univ. Grenoble Alpes, GIPSA-Lab, Grenoble F-38000,

CNRS, GIPSA-Lab, Grenoble F-38000

e-mail: jerome.mars@gipsa-lab.grenoble-inp.fr) 


\section{Introduction}

Various applications make use of arrays of hydrophones to estimate the direction of arrival (DOA) of underwater acoustic sources, including military surveillance, bioacoustics, and environmental monitoring, to name but a few. Such estimations can be made through two broad classes of methods:

1. Time-difference-of-arrival (TDOA) estimation.

2. Space-time processing for high-resolution estimation of the DOA, like beamforming [1] or spatial spectral estimation (Capon, MUSIC [2], ESPRIT [3, 4], and others).

The first class of methods is commonly used for bio-acoustic and environmental applications, such as the localization of cetaceans $[5,6,7,8]$, and it generally uses a small number of hydrophones (i.e., from 2 to 10). The estimation of the TDOA, which consists of the determination of the travel-time of the wavefront between two spatially separated receivers, relies generally on the cross-correlation, which is classical and easy-to-use, even for non-specialists in signal processing. Moreover, the cross-correlation gives good performances when the signal-to-noise ratio (SNR) is high and the useful signal is slightly distorted. Thorough state-of-the-art reviews of cross-correlation-based TDOA estimators can be found in $[8,9,10]$.

The second class of methods is mostly used when the array has a much greater number of receivers, and it is mostly used in applications such as SONAR [11], acoustical tomography [12], seismic-wave analysis [13], and digital communication. Methods from this class consist of the construction of a spatial spectrum by virtually steering the array in various directions and estimating the received power. When the array is steered in the direction of a source, the power received by the hydrophone array is maximized, whereas in the directions where no sources radiate, the received power is weak $[1,3,4,14]$.

In this article, we focus on the first class of methods, and more particularly, on estimation of the TDOA of acoustic signals with low SNR and strong distortions (when classical methods fail). Estimating the TDOA correctly is usually a challenging task, because underwater acoustic sources are immersed in a noisy environment and the propagation through the oceanic canal significantly modifies the signal by introducing amplitude and phase modulations. Thus, the signal received can be very different from one hydrophone to another, and only a few series of samples will remain similar on all of the hydrophones. After propagation, the received signals can be expressed as follows:

$$
\left\{\begin{array}{l}
s_{1}(t)=A_{1}(t) x(t)+n_{1}(t) \\
s_{2}(t)=A_{2}(t) x(t+T D O A)+n_{2}(t)
\end{array}\right.
$$

where $x(t)$ is the unknown source signal, $A_{1}(t)$ and $A_{2}(t)$ are random amplitude modulations introduced during the propagation between the source and each hydrophone, and $n_{1}(t)$ and $n_{2}(t)$ are uncorrelated additive white Gaussian noise (WGN). 
Based on these observations, we propose a new approach to estimate the TDOA that is derived from the field of nonlinear analysis of dynamical systems. Our estimator relies on cross-recurrence plots (CRPs) $[15,16]$, which are used to identify series of samples that are similar within the signals received by two hydrophones. The TDOA is then estimated from the CRP using some specific measures, called recurrence quantification analysis (RQA), which are especially dedicated to quantification of the information contained in CRPs. In this article, we consider six different RQA measures to estimate the TDOA. Four of these RQA measures come from the existing CRP literature $[17,18]$. However, we will show that classical RQA measures are not appropriate for TDOA estimation. Therefore, in section 2.2, we propose two new RQA measures that combine both the information contained in the CRPs and in the distance plots (or unthresholded CRPs). These latter RQA measures appear to be more appropriate for our purpose.

Cross recurrence plots have already been used for time-scale alignment of data series [16], to find nonlinear interrelations from bivariate time series with applications in climatology [17, 19], and to study synchronization of dynamic systems [18]. To our knowledge, this is the first time that CRPs are used with acoustic signals for TDOA estimation.

Section 2 recalls the basics of CRPs. Then some quantification measures are proposed to estimate the TDOA from the CRP. Section 3 tests the proposed method on simulated signals, and discusses the influence of the parameters involved in the CRP on the performance of the estimator. The proposed method is finally validated in section 4 using real bio-acoustic signals recorded at sea by a hydrophone array. This validation set shows that our method gives an increase of the correctly estimated TDOAs of $7 \%$ with respect to the classical cross-correlation estimator.

\section{Method}

\subsection{About Cross-Recurrence Plots}

Cross-recurrence plots are an extension to the bi-variate case of the recurrence plot analysis (RPA) from Eckman [20], which was later introduced by Zbilut et al [15], and then described with a mathematical formalism by Marwan et al [16]. CRPs are used to study the similarities and dependencies between two different complex systems, by comparisons of their respective states [16]. Three steps are involved to transform a data series from two systems to a CRP representation.

Considering two measured signals (denoted $x$ and $y$ ) recorded on two different sensors, the first step aims at the simultaneous reconstruction of the trajectory of each signal in the same phase space using the time-delay embedding method [21, 22]. The same embedding dimension $m$ and delay $\tau$ are used for both signals. The trajectory of each signal in the phase space domain is described by a series of phase space vectors given by: 


$$
\begin{gathered}
\overrightarrow{x_{m}(i)}=[x(i), x(i+\tau), \ldots, x(i+(m-1) \tau)] \\
\overrightarrow{y_{m}(j)}=[y(j), y(j+\tau), \ldots, y(j+(m-1) \tau)]
\end{gathered}
$$

where $i=\left\{1,2, \ldots, N_{x}-(m-1) \tau\right\}$ and $N_{x}$ are the sample index and the total number of samples of the measured signal $x$, respectively, and $j=\left\{1,2, \ldots, N_{y}-(m-1) \tau\right\}$ and $N_{y}$ are the sample index and the total number of samples of the measured signal $y$, respectively. To simplify the notations, we will consider that $x$ and $y$ have the same length $N=N_{x}=N_{y}$.

The second step consists of the measurement of the degree of similarity between the phase space vectors $\overrightarrow{x_{m}(i)}$ and $\overrightarrow{y_{m}(j)}$ associated to the two measured signals. The calculation of the similarities between all of the possible pairs of phase space vectors gives the similarity matrix with dimension $N \times N$ defined by:

$$
d(i, j)=\operatorname{Sim}\left(\overrightarrow{x_{m}(i)}, \overrightarrow{y_{m}(j)}\right)
$$

where $\operatorname{Sim}(.$, . $)$ is the function that is chosen to study the likeness of the phase space vectors. A lot of different mathematical functions can be used for this step [23]. The Euclidean norm is mostly used for this purpose by the recurrence plot community [18]. However, the Euclidean norm is not suitable for our application, because it is not adapted to the strong amplitude differences that exist between the noise samples and the useful signal samples. Using the Euclidean norm leads to finding similarities between vectors associated to the noise, because the noise samples generally have low amplitude, which leads to low Euclidean norm, although this also leads to finding similarities between vectors associated to the vectors of useful signals that look alike and where their Euclidean norms are also close to zero.

Instead, we prefer to use the dot product and Pearson's correlation coefficient (PCC), which are both particular cases of the cross-correlation classically used for TDOA estimation. These last two similarity functions are more appropriate to our signals because they both give high values when phase space vectors of the useful signal look alike, while they give a value close to 0 when the vectors are not alike. Therefore, when thresholding the similarity matrix obtained with these similarity functions, we only get recurrences associated to the useful signal. We have already used the dot product and PCC as a similarity function in previous studies relative to the use of RPA for detection purposes [23]. These similarity functions have already proven to be very interesting solutions when studying various kinds of simulated and real signals with RPA [23, 27, 28].

The dot-product between two phase space vectors is given by:

$$
d_{i, j}=\sum_{k=1}^{m} x_{k}(i) \times y_{k}(j)
$$

where $x_{k}(i)$ and $y_{k}(j)$ are the $k^{t h}$ components of vectors $\overrightarrow{x_{m}(i)}$ and $\overrightarrow{y_{m}(j)}$, respectively.

The PCC between two phase space vectors is given by: 


$$
d_{i, j}=\frac{\sum_{k=1}^{m}\left(x_{k}(i)-\overline{x_{k}(i)}\right)\left(y_{k}(j)-\overline{y_{k}(j)}\right)}{\sqrt{\sum_{k=1}^{m}\left(x_{k}(i)-\overline{x_{k}(i)}\right)^{2}} \sqrt{\sum_{k=1}^{m}\left(y_{k}(j)-\overline{y_{k}(j)}\right)^{2}}}
$$

where $x_{k}(i)$ and $y_{k}(j)$ are the $k^{t h}$ components of vectors $\overrightarrow{x_{m}(i)}$ and $\overrightarrow{y_{m}(j)}$, respectively, and $\overline{x_{k}(i)}$ and $\overline{y_{k}(j)}$ are the empirical means of $\overrightarrow{x_{m}(i)}$ and $\overrightarrow{y_{m}(j)}$, respectively.

Finally, in the last step, the CRP is obtained by comparing each coefficient of the similarity matrix to a threshold $\varepsilon$. Therefore, the CRP is a binary matrix, where the coefficient of index $(i, j)$ is 1 if $\overrightarrow{x_{m}(i)}$ and $\overrightarrow{y_{m}(j)}$ are considered as similar, and it is 0 otherwise. In mathematical formalism, the CRP is defined as follows:

$$
C R P(i, j)=\Theta\left(\operatorname{Sim}\left(\overrightarrow{x_{m}(i)}, \overrightarrow{y_{m}(j)}\right)-\varepsilon\right)
$$

where $\Theta$ is the Heaviside function.

\subsection{Recurrence Quantification Analysis}

When calculating the CRP of signals received by two spatially separated sensors, a recurrence pattern shows up on the binary image obtained. As shown in Figure 1, the recurrence pattern, which is composed of 15 parallel diagonal lines in our example, is only related to the useful signal (cosine function) and not to the noise. The noise is not supposed to produce any recurrence pattern. The position of the recurrence pattern of the useful signal depends on the TDOA. For example, in Figure 1, receiver 1 is taken as the reference and three different TDOAs are simulated by translation of the signal of receiver 2 from -70 samples to +70 samples. These translations of the cosine on receiver 2 lead to horizontal translation of the associated recurrence pattern on the CRP (Fig. 1). If receiver 2 was taken as the reference and the cosine was translated on receiver 1 , then the recurrence pattern associated to the cosine would be translated vertically on the CRP.

The automatic quantification of this pattern, like the diagonal or vertical structures, and of its position, can be performed using the measures known as RQA $[24,25,26]$. The RQA measures can be computed on either the entire CRP or separately on each diagonal parallel to the main diagonal $[17,18]$.

For the TDOA estimation we want to perform in this paper, we use these latter modified RQA measures, which are calculated for each diagonal of the CRP taken separately, and are therefore based on recurrences that form diagonal lines parallel to the main diagonal $[17,18]$.

Relying on notations introduced in [17], the RQA measures are expressed as a function of the index of the diagonal $t \in[-T, \ldots, T]$, where $t=0$ corresponds to the main diagonal, $t>0$ are the diagonals above the main diagonal, and $t<0$ are the diagonals below the main diagonal. $T$ is the maximum allowable TDOA (expressed as samples) with respect to the geometry of the array. Diagonals with 


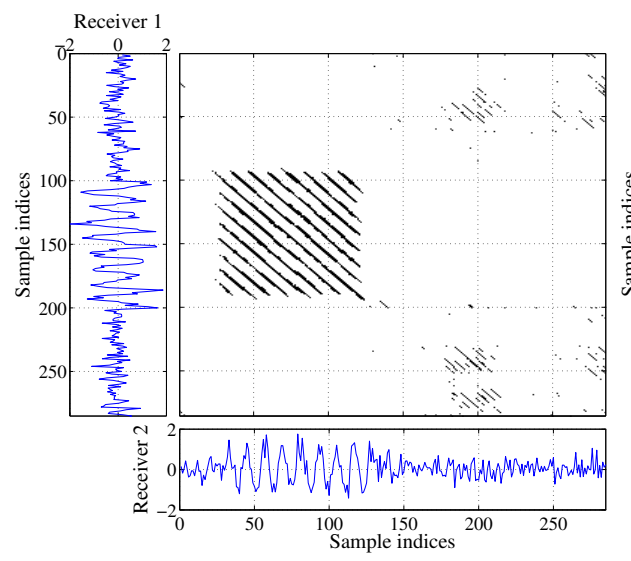

(a) $\mathrm{TDOA}=-70$ samples

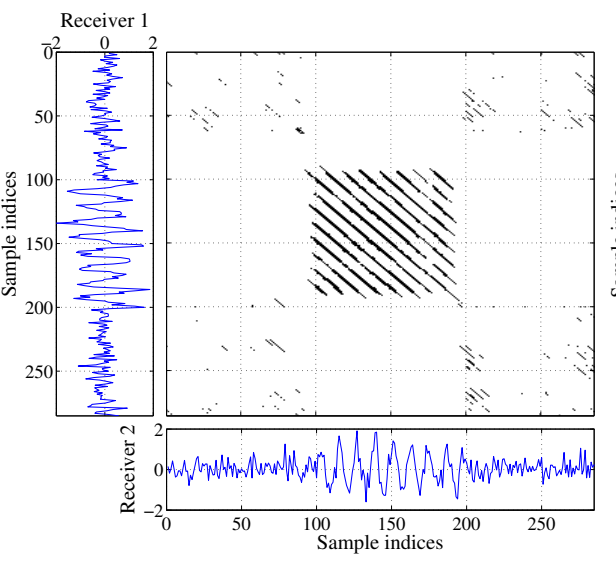

(b) $\mathrm{TDOA}=0$ samples

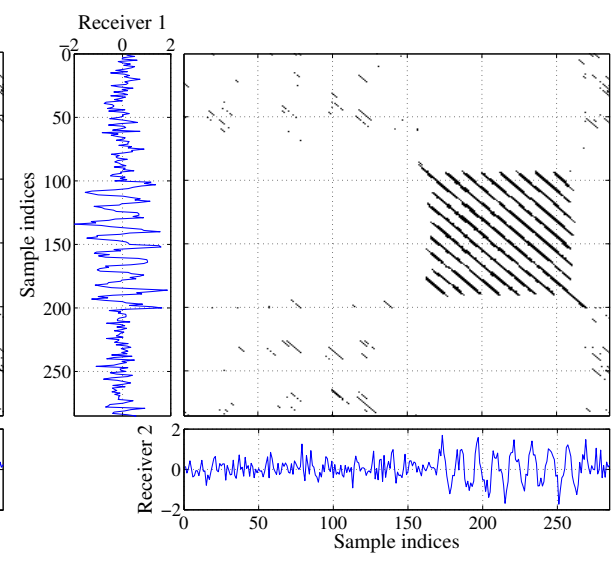

(c) $\mathrm{TDOA}=+70$ samples

Fig. 1: Examples of CRP matrices in the case of a 100-sample cosine mixed with 300-sample additive WGN received by two sensors with three different TDOAs: (a) TDOA = -70 samples; (b) TDOA $=0$ samples; (c) TDOA $=+70$ samples. The SNR is $6 \mathrm{~dB}$. The $\mathrm{CRP}$ is built with $\mathrm{m}=16, \tau=1, \operatorname{Sim}(.$, . .) $=$ dot product, $\varepsilon=0.075$.

indices $t>0$ represent the positive TDOAs, while diagonals with indices $t<0$ represent the negative TDOAs.

We recall the expressions of the RQA measures from the literature [17, 18], and we introduce two new RQA measures that we consider for the TDOA estimation. The first four RQA measures from the literature only rely on the length of the diagonal lines of the CRPs, and so they are based only on the binary information contained in the CRPs. We will show that these RQA measures have some limitations and are not appropriate for our purpose. To overcome these limitations. we propose two new RQA measures, which combine the information from the CRP with that of the distance matrix, and which leads to more accurate results.

The first RQA measure is the average diagonal line length given by:

$$
L(t)=\frac{\sum_{l=l_{\min }}^{N-|t|} l P_{t}(l)}{\sum_{l=l_{\min }}^{N-|t|} P_{t}(l)}
$$

where $P_{t}(l)$ is the distribution of diagonal line lengths for diagonals with index $t$, $l_{\min }$ is the threshold that sets the length of the shortest diagonal line considered, and $N$ is the total number of lines (resp. columns) of the CRP matrix. This RQA measure characterizes the duration of similarities between both signals [17]. Thus, for the diagonal with index $t$ associated to the real TDOA value, we expect that the diagonal lines are long, so that we reach a global maximum for $L(t)$ that indicates 
that the highest coincidence between both signals is reached for this specific delay $t$.

The second RQA measure is the determinism given by:

$$
\operatorname{DET}(t)=\frac{\sum_{l=l_{\min }}^{N-|t|} l P_{t}(l)}{\sum_{l=1}^{N-|t|} l P_{t}(l)}
$$

As for the average diagonal line length $L(t)$, the determinism $D E T(t)$ characterizes the proportion of recurrence points that form long diagonal structures of all of the recurrence points. A high determinism for the diagonal $t$ indicates that both signals have similar dynamic behavior over a long time span with a certain delay $t$ [17]. Therefore, we expect that the determinism will reach a global maximum for the diagonal with index $t$ associated to the TDOA that we try to estimate.

The third RQA measure is the longest diagonal line within diagonal $t$, given by:

$$
L_{\max }(t)=\underset{i \in\left\{1, \ldots N_{l}\right\}}{\arg \max }\left(l_{i}\right)
$$

where $i \in\left\{1, \ldots N_{l}\right\}$ is the index of the diagonal line, and $N_{l}$ is the total number of diagonal lines within diagonal $t$. As above, we expect to get a global maximum for $L_{\max }(t)$ at the index $t$ associated to the TDOA.

The fourth RQA measure is the recurrence rate, which is defined as:

$$
R R(t)= \begin{cases}\frac{1}{N+t} \sum_{i=1}^{N+t} C R P(i-t, i) & (t<0) \\ \frac{1}{N-t} \sum_{i=1}^{N-t} C R P(i, i+t) & (t \geq 0)\end{cases}
$$

where $N$ is the total number of lines (resp. columns) of the CRP matrix. This RQA measure gives the probability of occurrence of similar waveforms in both signals with a certain time delay $t$ [17]. The greater the number of recurrences in the diagonal $t$ is, the greater the value of $R R(t)$ is. Again, we expect that $R R(t)$ reaches a global maximum for the diagonal $t$ associated to the TDOA value.

$R R(t)$ only relies on the binary matrix obtained with the CRP, and does not take into account all of the information contained in the similarity matrix $d(i, j)$ given by Equation (4) that might be valuable. Therefore, we introduce two additional RQA measures that also rely on the similarity matrix $d(i, j)$.

The first proposed RQA measure is the sum of all similarity values $d(i, j)$ from the diagonal $t$ that have led to recurrence points (i.e., $\operatorname{CRP}(i, j)=1$ ) in the CRP matrix. This is defined as: 


$$
S S(t)= \begin{cases}\sum_{i=1}^{N+t} C R P(i-t, i) \odot d(i-t, i) & (t<0) \\ \sum_{i=1}^{N-t} C R P(i, i+t) \odot d(i, i+t) & (t \geq 0)\end{cases}
$$

where $\odot$ is the Hadamard product.

The last proposed RQA measure is a normalized version of the previous one, and it is given by:

$$
S R(t)=\left\{\begin{array}{l}
\frac{1}{N+t} \sum_{i=1}^{N+t} C R P(i-t, i) \odot d(i-t, i) \quad(t<0) \\
\frac{1}{N-t} \sum_{i=1}^{N-t} C R P(i, i+t) \odot d(i, i+t) \quad(t \geq 0)
\end{array}\right.
$$

As for $R R(t), S S(t)$ and $S R(t)$ are high if the number of recurrences in diagonal $t$ and the associated coefficients in the similarity matrix are simultaneously both high. A global maximum is expected for $R R(t), S S(t)$, and $S R(t)$ when $t$ is equal to the TDOA to estimate.

\subsection{Time-Difference-of-Arrival Estimated with RQA Measures}

For the six RQA measures presented in the previous section, we said that their values would be high if both signals have similar waveforms with a certain delay $t$. Therefore, the estimated TDOA is obtained by looking for the diagonal $t$ that gives the global maximum of each RQA measure. This is given by:

$$
\widehat{T D O A}=t \text { such that } \underset{t}{\operatorname{argmax}}(R Q A(t))
$$

where $t \in[-T, \ldots, T]$ and $R Q A(t)$ is either $L(t), D E T(t), L_{\max }(t), R R(t), S S(t)$ or $S R(t)$.

\section{Results on Simulated Data}

In this section, we use simulated signals to study the reliability of CRPs and RQA measures as TDOA estimators. We will show that the RQA measure $S S(t)$ is the most appropriate one. We will also discuss the influence of the embedding dimension $m$, of the similarity function $\operatorname{Sim}(.,$.$) , of the SNR and the TDOA value to esti-$ mate, on the overall performances of the proposed method. Finally, we will compare the proposed method with the cross-correlation, which is classically used for TDOA estimation in several fields. 


\subsection{Performance Analysis Methodology}

To analyze the performances of the proposed method, we use simulated signals as they would be received independently by two sensors. The characteristics of these simulated signals are representative of the real acoustic signals used in section 4 . The received signals are cosine functions of frequency $f_{0}$, where their respective amplitudes $A_{1}(t)$ and $A_{2}(t)$ are modulated randomly according to a first-order autoregressive model. The WGN denoted as $n_{1}(t)$ and $n_{2}(t)$ is also added to the two receivers, to achieve a certain SNR. A time-delay is added to the second signal to model the TDOA. Therefore, the two simulated signals can be written as follows:

$$
\begin{gathered}
s_{1}(t)=A_{1}(t) \times \cos \left(2 \pi f_{0} t\right)+n_{1}(t) \\
s_{2}(t)=A_{2}(t) \times \cos \left(2 \pi f_{0}(t+T D O A)\right)+n_{2}(t)
\end{gathered}
$$

We can recall that a first-order autoregressive model is given by:

$$
A(t)=\alpha A(t-1)+Y(t)
$$

where $|\alpha| \in[0,1[$ so that the process remains stationary in a wide sense, and $Y(t)$ is WGN with zero mean and variance of 1 .

For the simulations, we take $f_{0}=1 \mathrm{kHz}$, which is the frequency of the real acoustic signal used in section 4 , and a sampling frequency $f_{e}=12 \mathrm{kHz}$. The SNR varies as follows: $\{-7 ;-5 ;-3 ; 0 ; 3 ; 5 ; 7 ; 10\} \mathrm{dB}$. For the autoregressive model, we arbitrarily choose $\alpha=0.98$, so that the integration time of the process is about nine cosine periods. Moreover, the peak amplitude of the autoregressive model is normalized to the following values: $A_{\text {peak }}=\{0.4 ; 0.7 ; 1 ; 1.3 ; 1.5 ; 1.9\}$, to reach various modulation indices. Figure 2 shows an example of the simulated signal as received by the two sensors, with various SNRs.

We study the performances of the proposed estimator for five different theoretical TDOA values; namely $[0,4,15,40,100]$ samples. This is to determine the reliability of the method when the TDOA corresponds to the diagonals that are either close to or far from the main diagonal of the CRP matrix, and also when the TDOA is lower or higher than the period of the cosine signal.

For each set of sextuple $\left(m, \varepsilon, \operatorname{Sim}(.,),. S N R, A_{\text {peak }}, T D O A\right)$ we repeat the simulations 500 times, to derive a statistical analysis of the results. The performances are assessed by calculation of the ratio of correct estimates over the total number of estimates. An estimated TDOA is considered as correct if it is exactly equal to the simulated TDOA.

\subsection{Performances of the RQA Measures}

In this section, we study the relevance of the proposed RQA measures as tools for TDOA estimation. We want to identify the RQA measures that appear to be appro- 

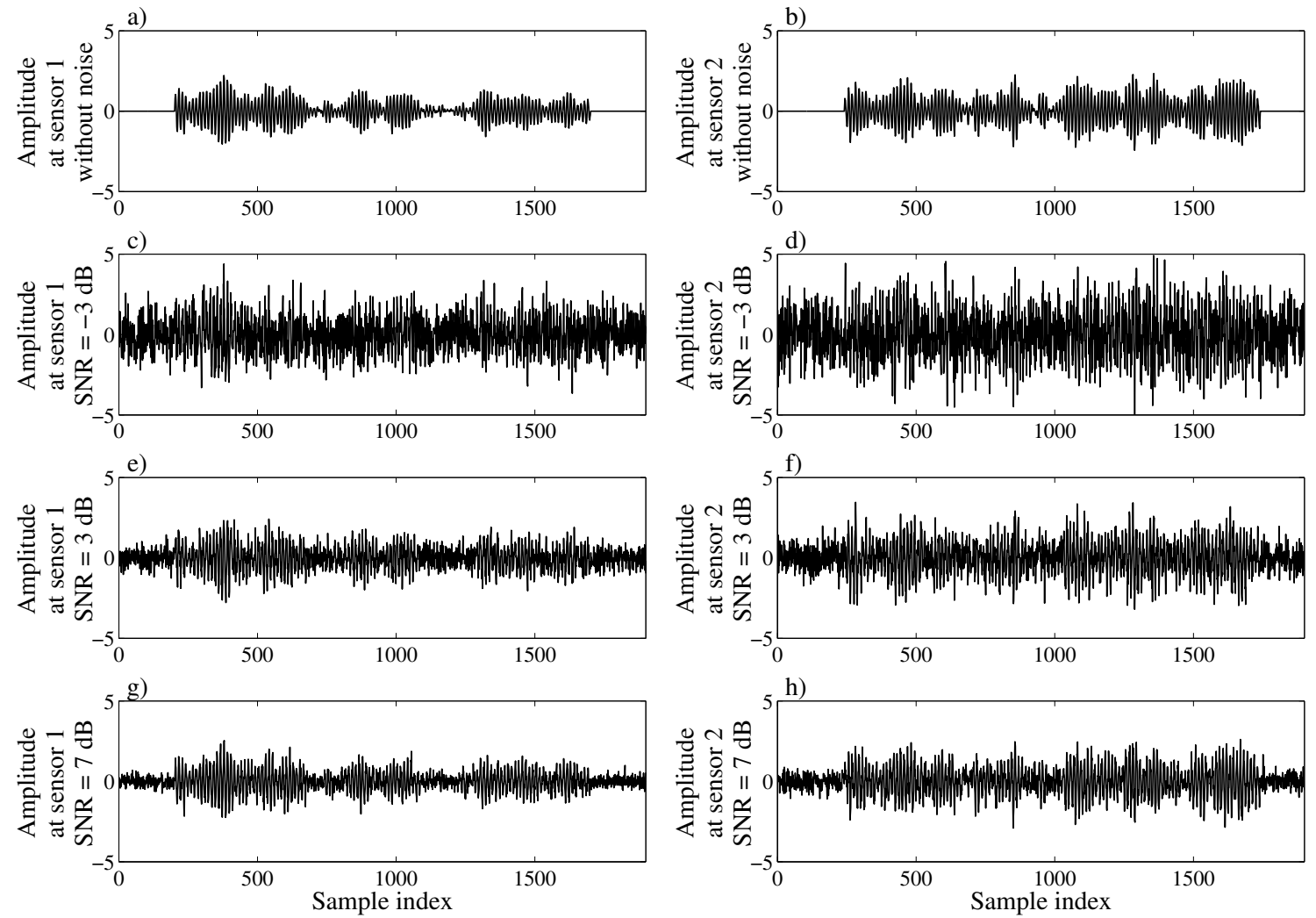

Fig. 2: $(a, b)$ Simulated signals received by two sensors without additional noise. Each of these is a cosine function where the amplitude is modulated by a first-order auto-regressive model, the peak amplitude of which is $A_{\text {peak }}=1.5$. (c, d) Simulated signal added to WGN with SNR $=-3 \mathrm{~dB}$. (e, f) Simulated signal added to WGN with $\mathrm{SNR}=3 \mathrm{~dB}$. (g, h) Simulated signal added to WGN with SNR $=7 \mathrm{~dB}$.

priate for our TDOA estimation problem, and to discard the other ones. We will show that the two proposed RQA measures outperform the classical ones.

Figure 3 shows the results of the simulations for three sets of parameters; namely $\tau=1, m=16, \operatorname{Sim}(.,)=.P C C$, a recurrence threshold $\varepsilon$ giving a fixed recurrence point density of $10 \%$ over the entire CRP, $A_{\text {peak }}=1$, and TDOA $=0, T D O A=15$ and $T D O A=40$. However, the conclusions remain the same whatever the variations of the parameters $m, \varepsilon, S N R, T D O A$ and $A_{\text {peak }}$.

We notice that the RQA measure $S S$ (Fig. 3, solid line) always gives the best performances, as its percentage of correct estimates is always the highest, whatever 
the SNR, TDOA, similarity function $\operatorname{Sim}(.$, . .), and amplitude of the modulations $A_{\text {peak }}$.

The RQA measure $S R$ (Fig. 3, line with squares) follows the same trend as $S S$, but with a lower performance. All of the other proposed RQA measures, i.e., $L, L_{\max }$, $D E T$, and $R R$, give very poor results and are not suitable for the TDOA estimation. A detailed observation of the simulation results shows that these four latter RQA measures give estimates that are far from the true simulated TDOAs.

Finally here, it is important to note that the percentage of correct estimates obtained with $S S$ is independent of the TDOA, which is not the case with SR. For example, the plots given in Figure 3 show that for $\mathrm{SNR}=7 \mathrm{~dB}$ the RQA measure $S S$ gives a percentage of correct estimates of about $69 \%( \pm 1 \%)$ for all three TDOA values, whereas $S R$ gives a percentage of correct estimates of about $30 \%( \pm 10 \%)$. These observations and conclusions remain valid for other simulated values of amplitude modulations $A_{\text {peak }}$ (not presented here). Thus, $S S$ gives the most consistent results for all of the sets of parameters, and it is therefore the most reliable RQA measure to estimate TDOAs.

According to these results, in the remainder of this article we only use the RQA measure SS, as defined by Equation (12) to estimate TDOAs, and all of the results will now be given with this particular RQA measure.

a) Theoretical TDOA $=0$

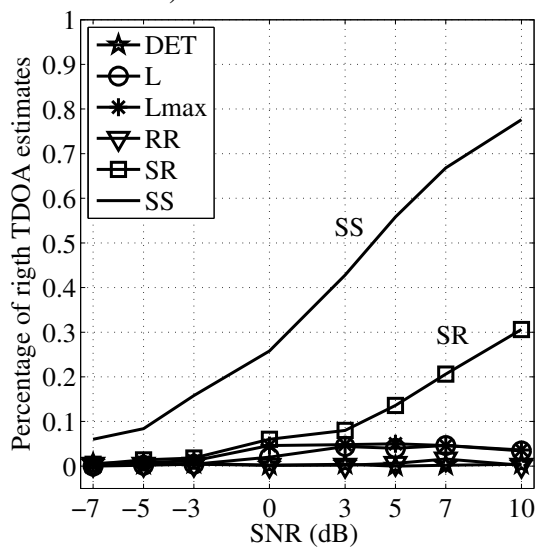

b) Theoretical TDOA $=15$

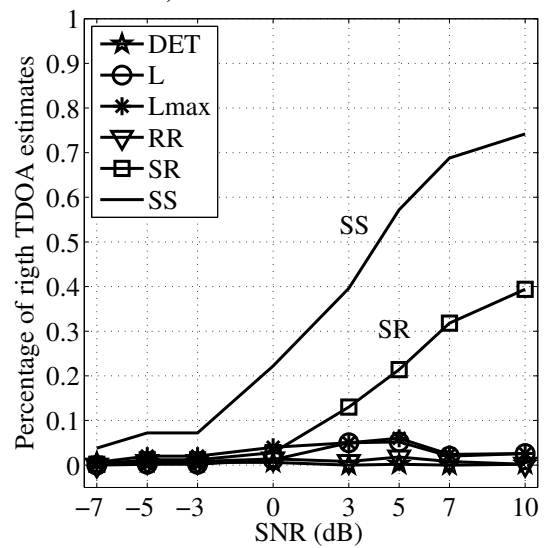

c) Theoretical TDOA $=40$

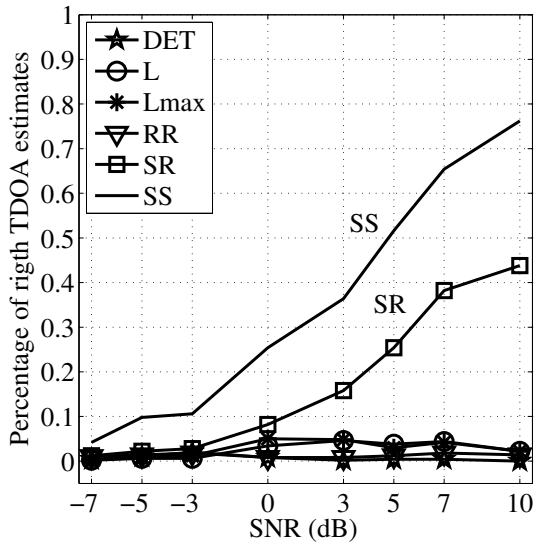

Fig. 3: Percentages of good estimates over the total number of estimates as a function of the SNR of the RQA measure and for three simulated TDOAs: (a) TDOA= 0 ; (b) TDOA $=15$; (c) TDOA $=40$. The parameters of the simulations are: $\tau=1$, $m=16, \operatorname{Sim}(.,)=.P C C, A_{\text {peak }}=1$. The RQA measure $S S$ almost always gives the highest percentages, and it is therefore the most appropriate RQA measure for our TDOA estimator. 


\subsection{Performances as a Function of the Parameters Used to Build the CRP}

In this section, we study the influence of the similarity function $\operatorname{Sim}(.,$.$) , of the$ embedding dimension $m$, and of the recurrence threshold $\varepsilon$ used to compute the CRP on the performances of the TDOA estimator.

\subsubsection{Influence of the Embedding $m$}

Simulations are performed for numerous embedding dimension values within the range of 4 to 24 , while keeping $\tau=1$ constant, and $\varepsilon$ so that the recurrence point density over the entire CRP is always $10 \%$. Figure 4 summarizes the results of these simulations for a given set of parameter: $\left(A_{\text {peak }}=1.5, T D O A=100, R Q A=S S\right)$. The conclusions remain similar for all of the other sets of parameters, whatever the values given to $A_{\text {peak }}$ and $T D O A$.

We can see in Figure 4a that if the similarity function is the PCC, the percentage of correct estimates increases when $m$ increases. When $m$ increases from 4 to 10 in particular, the percentage of correct estimates is multiplied by three on average (which depends on the SNR), while when $m$ increases from 12 to 24, the percentages of correct estimates increases by just a few percent. For example, from $m=12$ to $m=24$, the percentage of correct estimates increases only by about $15 \%$, whatever the SNR.

If the similarity function is the dot product, then the percentage of correct estimates is very similar for all of the embedding dimensions (Fig. 4b).

\subsubsection{Influence of the Similarity Function}

For this subsection, we keep the parameters $\tau, m$, and $\varepsilon$ used to build the CRP constant, and we study the influence of the similarity function on the performances of the proposed estimator. Simulations are carried out for various combinations of TDOA and of amplitude modulations $A_{\text {peak }}$. The most significant results are shown in Figure 5.

We note that when $A_{\text {peak }}=0.4$ (Fig. 5, first row of the panel), the estimator using the dot product always gives a better percentage of correct estimates than the estimator with the PCC, whatever the SNR and the TDOA. When $A_{\text {peak }}=1$ (Fig. 5, second row of the panel), both similarity functions give very similar results, whatever the SNR and the TDOA. When $A_{\text {peak }}=1.5$ (Fig. 5, third row of the panel), the estimator with the PCC has better performances than the one with the dot product.

By examination of the results of the simulations for all six simulated values of amplitude modulation, we can conclude that when the signals are weakly modulated, typically $A_{\text {peak }}<1$, then the estimator using the dot product always outperforms the estimator with the PCC. Then, as the signal is more and more modulated, 
a) $\operatorname{Sim}(.,)=$. Pearson correlation coefficient

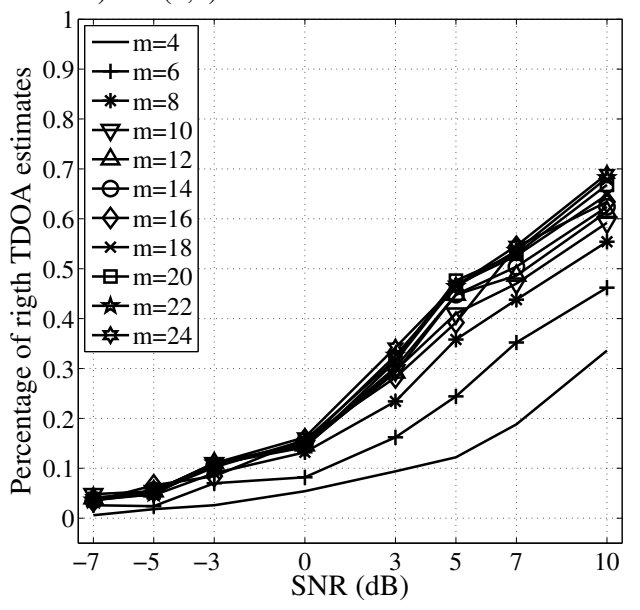

b) $\operatorname{Sim}(.,)=$. Dot product

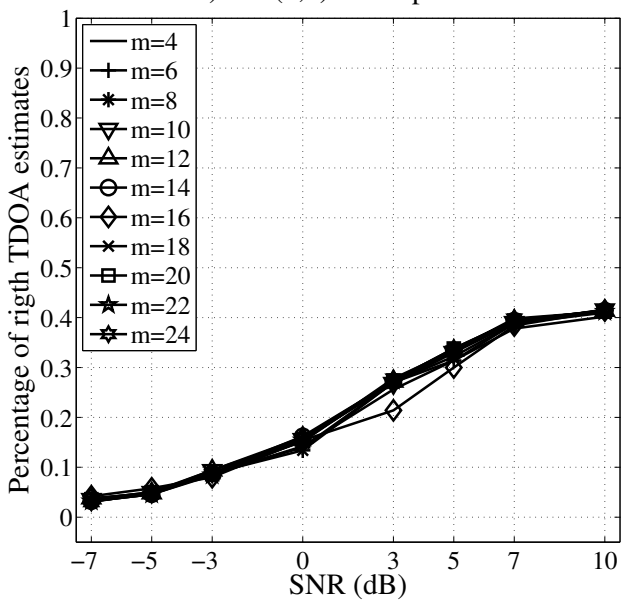

Fig. 4: Percentage of good estimates over the total number of estimates as a function of the embedding dimension $m$, of the SNR, and of the similarity function $\operatorname{Sim}(.,$.$) :$ (a) $\operatorname{Sim}(.,)=.P C C$; (b) $\operatorname{Sim}(.,)=$. Dot product. The parameters of the simulations are: $\tau=1, A_{\text {peak }}=1.5, T D O A=100$ and RQA measure $S S$.

i.e., the $A_{\text {peak }}$ increases, the performances of the estimator using the dot product decreases. Similar performances are obtained with both estimators when $A_{\text {peak }}=1$. Finally, for highly modulated signals with a lot of fading (i.e., $A_{\text {peak }}>1$ ), the estimator using PCC always outperforms the estimator with the dot product.

Whatever the similarity function used, when $A_{\text {peak }}$ increases, the percentage of correct estimates decreases. However, the rate at which performances decrease is not the same for all of the estimators. If the dot product is used, then for all SNRs the percentages of correct estimates are divided by a factor of about 2.5 when $A_{\text {peak }}$ goes from 0.4 to 1.5 . At the same time, the percentages of correct estimates are divided by a factor of at most 1.5 if the estimator uses the PCC.

Relying on these observations and conclusions, we will choose the PCC as a similarity function to estimate the TDOA on the real acoustic data used in section 4 .

\subsubsection{Influence of the Recurrence Threshold}

The choice of the recurrence threshold affects the number of recurrence points that appear on the CRP. Simulations are made by keeping $\tau$ and $m$ constant, while the values of $\varepsilon$ are adjusted so that they give a fixed recurrence point density over the entire CRP. Performances are studied for recurrence thresholds with the following recurrence densities: $1 \%, 2 \%, 5 \%, 8 \%, 10 \%, 12 \%, 15 \%, 20 \%$ and $25 \%$. These simulations show that for a given set of values for the quintuple ( $\tau, m, A_{\text {peak }}, T D O A$, $S N R)$, the percentage of correct estimates is nearly the same for all of the recurrence 

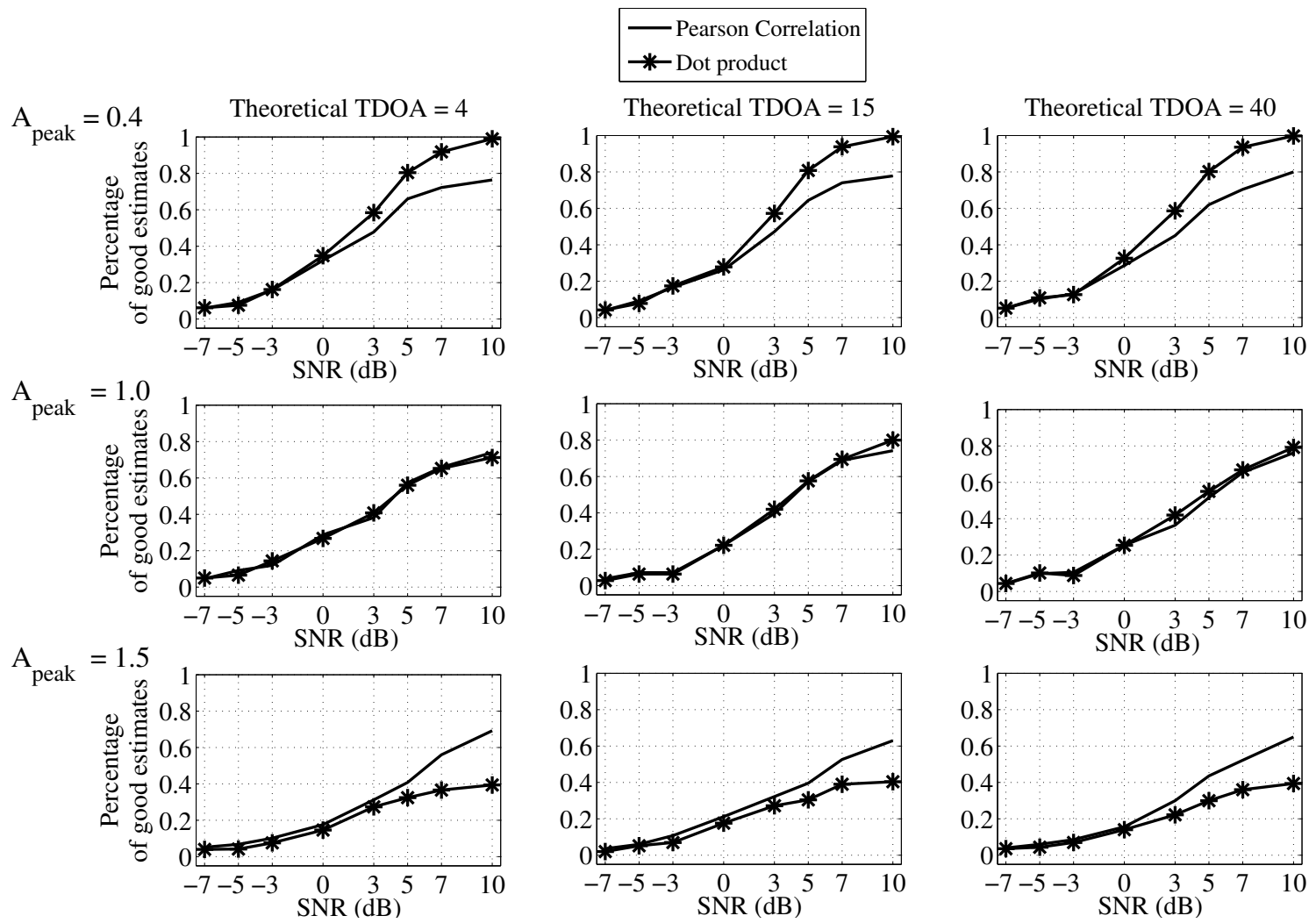

Fig. 5: Percentage of correct estimates over the total number of estimates as a function of the SNR, of the TDOA (each column of the panel), and of the amplitude of the modulation $A_{\text {peak }}$ (each line of the panel). The results with $\operatorname{Sim}(.,)=.P C C$ are shown with the solid line. The results with $\operatorname{Sim}(.,)=$. Dot Product are shown with the stars $(*)$. The parameters of the simulations are: $\tau=1, m=16$, and RQA measure $S S$.

densities used. Figure 6 shows an example of these results when $\tau=1, m=12$, $A_{\text {peak }}=1.5, T D O A=40, S N R \in[-7 ; 10] \mathrm{dB}$. Therefore, we can conclude that our method is invariant with regard to the recurrence threshold.

\subsection{Comparison with the Classical Cross-Correlation}

In this last section, we compare the performances of the proposed estimator with an estimator based on the cross-correlation, which is classically used for this purpose. Unlike the CRP, which divides the signal into multiple smaller pieces that are repre- 


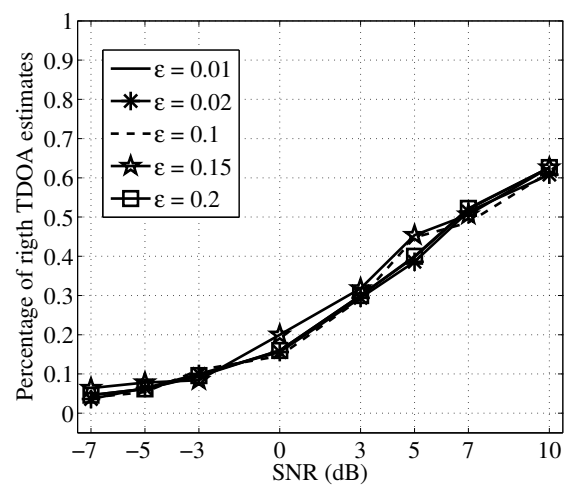

Fig. 6: Percentages of correct estimates over the total number of estimates as a function of the SNR and the recurrence densities: $1 \%, 2 \%, 10 \%, 15 \%$ and $20 \%$. The parameters of the simulations are: $\tau=1, m=12, A_{\text {peak }}=1.5, T D O A=40$, $\operatorname{Sim}(.,)=.P C C$, and RQA measure $S S$.

sented by the phase space vectors to estimate the TDOA, the cross-correlation uses the whole signal at once and the estimated TDOA is then the lag associated to the global maximum of the cross-correlation function. All of the previous simulations were performed with the cross-correlation as the TDOA estimator.

The results obtained here are shown in Figure 7, and they are compared with those of the proposed estimator. If the received signals are weakly modulated, then the cross-correlation outperforms the CRP-based estimator with the PCC, whereas this latter outperforms the cross-correlation when signals are highly modulated. For a given TDOA, we note that the results of the cross-correlation are very dependent of the amplitude of the modulations, while for a given amplitude of the modulation, the results stay consistent for all simulated TDOAs. Comparing Figure 5 and Figure 7, we note that for a given triplet $\left(S N R, A_{\text {peak }}, T D O A\right)$, the cross-correlation gives exactly the same percentage of correct estimates as the CRP with the dot product as a similarity function.

\section{Validation on Real Data}

In the previous subsection, we studied the performances of a CRP-based TDOA estimator and looked at the influence of the parameters used to built a CRP representation using simulated signals. This helped us to define $S S$ as the most appropriate RQA measure to estimate the TDOA. We also showed that the PCC is the similarity function that gives the most consistent results for various conditions of the SNR and the modulation of the received signal. In this section, we validate the proposed 

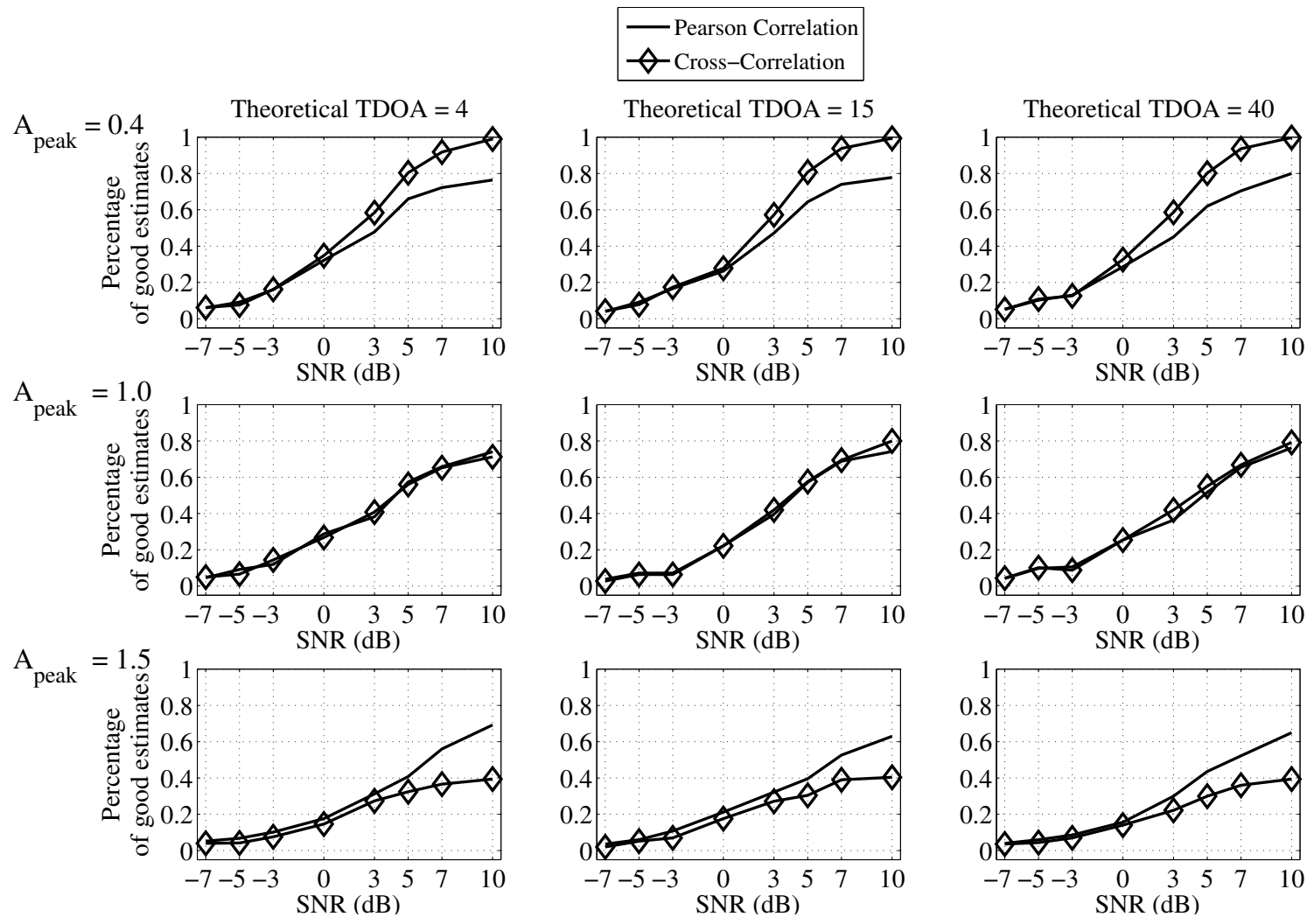

Fig. 7: Percentages of correct estimates over the total number of estimates as a function of the SNR, of the TDOA (each column of the panel), and of the amplitude of the modulation $A_{\text {peak }}$ (each line of the panel). The results of the proposed method with similarity function $\operatorname{Sim}(.,)=.P C C$ are given by the solid line. The results obtained with classical cross-correlation are shown with the diamonds $(\diamond)$. The parameters of the simulations are: $\tau=1, m=16$, and RQA measure $S S$.

TDOA estimator on real acoustic signals, and show again that it outperforms the classical cross-correlation.

\subsection{Material and Methods}

The database used in this section contains underwater sounds that were recorded in the Bay of Calvi (Corsica Island, France). The data were acquired by an autonomous acoustic recorder (RTSys EA-SDA14) that recorded the signals coming from four hydrophones (HTI-92, High Tech Inc., Gulport, MS, USA) simultane- 
ously, at a sampling frequency of $156.25 \mathrm{kHz}$. The data were digitized at 24 bits, and saved as WAV files on a hard drive. The four hydrophones form an array with a pyramidal shape. The distance between each hydrophone pair was about $1.5 \mathrm{~m}$. The antenna was placed on the seafloor at a depth of $38 \mathrm{~m}$ and the hydrophones were between $1 \mathrm{~m}$ and $1.5 \mathrm{~m}$ above the seafloor (Fig. 8).

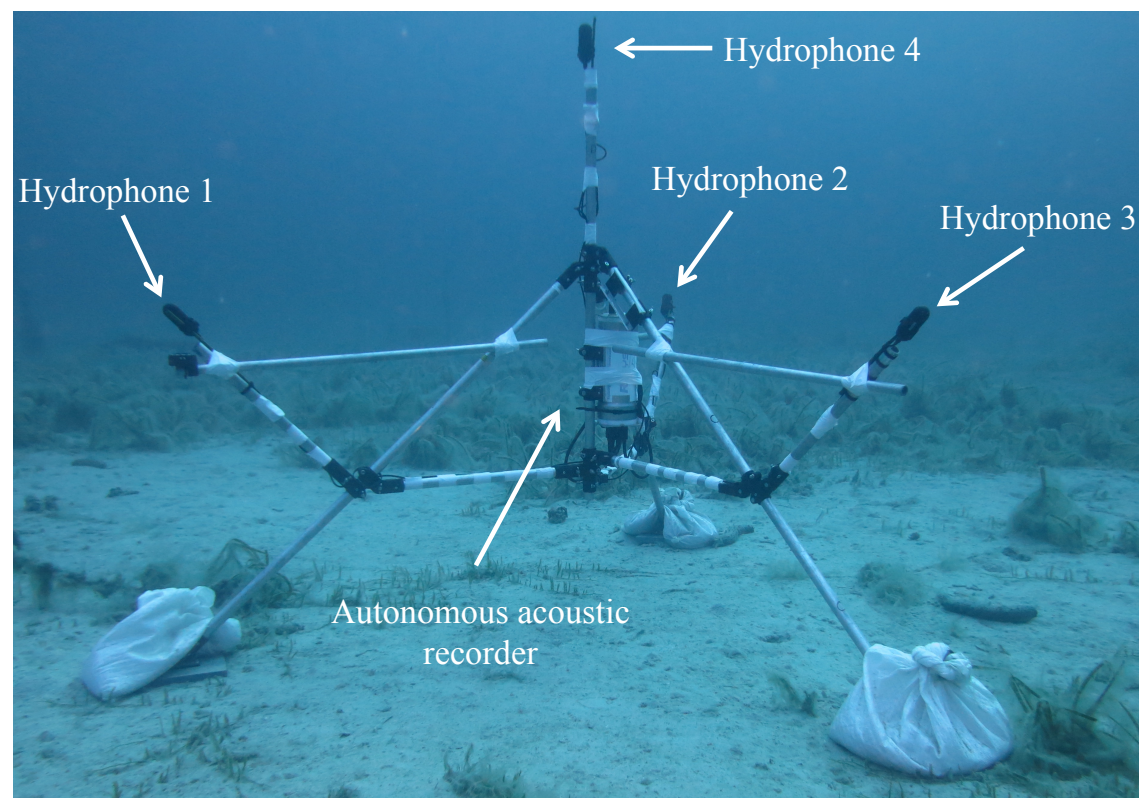

Fig. 8: Set-up of the antenna used to record the data. Four hydrophones are connected to four synchronized channels of an autonomous acoustic recorder.

The sounds of interest come from a Mediterranean fish of undetermined species, and they are short frequency modulated signals that last about $0.15 s$ and have a mean frequency of between $800 \mathrm{~Hz}$ and $1000 \mathrm{~Hz}$. Figure 9 shows two examples of these acoustic signals, from which we can clearly note that the amplitude modulations differ significantly from one hydrophone to another due to the effects of the propagation in the seawater. We manually annotated 56 of these sounds and then estimated the TDOAs between all of the possible pairs of hydrophones. As four hydrophones form six separate pairs, we obtained a total of 336 estimated TDOAs to validate the proposed TDOA estimator.

Prior to the TDOA estimation by our CRP-based method, the annotated sounds were down-sampled at $10 \mathrm{kHz}$ to reduce the size of the CRP matrix and to speed up the algorithm, and then they were bandpass filtered between $100 \mathrm{~Hz}$ and 2000 Hz. The CRP was then computed with the following parameters: $\tau=1, m=18$, $\operatorname{Sim}(.$, . $)=$ PCC, and a fixed threshold $\varepsilon=0.75$. The TDOAs were then estimated with the RQA measure $S S$ given by Equation (12). The TDOAs were also estimated 
with classical cross-correlation, and the results were compared to those of the CRPbased method, to determine whether this latter was more accurate and efficient.



b) Record 2
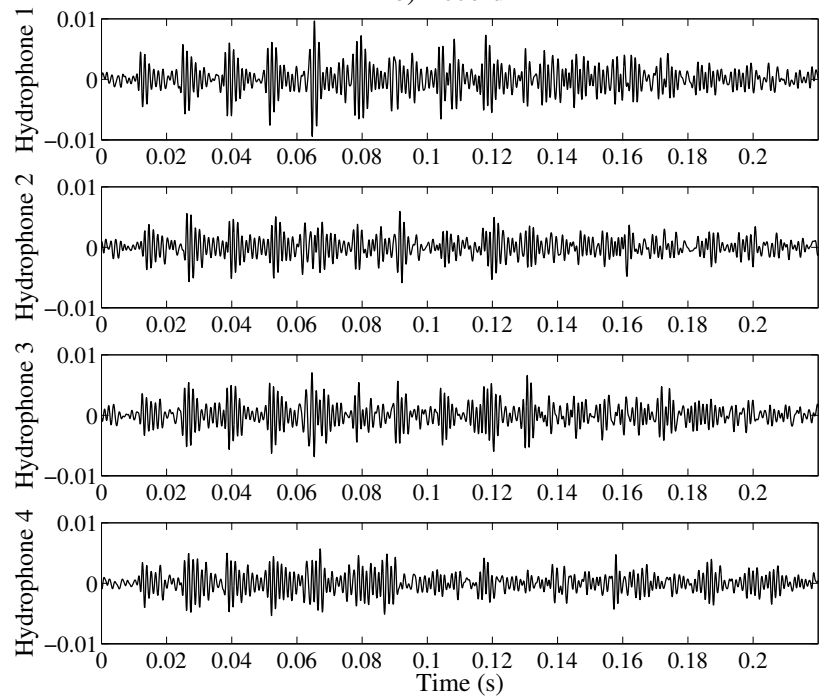

Fig. 9: Examples of two distinct recordings of the sounds produced by a fish (undetermined species) and recorded simultaneously by four hydrophones. 


\subsection{Methodology to Assess the Results on Real Data}

When using real data, we face the problem of knowing the ground truth to validate our results. To assess the validity of the estimated TDOAs given by our method and by the cross-correlation, we used both automatic and visual checking.

The automatic checking was based on the idea that each annotated signal gives six TDOA values, which are not independent of each other, but must respect transitive relations. For example, the TDOA between hydrophones 1 and 4 must be equal to the sum of the TDOA between hydrophones 1 and 2 and hydrophones 2 and 4 . Checking all of the possible transitive relations helped us to identify possible misestimated TDOAs. Details of the method we followed for this automatic checking can be found in the Appendix.

The visual inspection was performed by superimposing the waveform received by each pair of hydrophones and compensating for the TDOA between both signals with its estimated value. Because of the periodic nature of the received signals, we could quickly assess whether both signals were re-aligned correctly or whether the estimated TDOA was biased by a number of samples that are proportional to the oscillation period of the signal.

As an example, Figure 10a shows the signals received simultaneously by two hydrophones without compensating for the TDOA. It appears that these signals are not synchronized, as their respective waveforms are not superimposed. Figure 10b shows the same signals after the TDOA between the two hydrophones was compensated by the estimated value obtained with the proposed method (i.e., CRP with CPP and SS). We note that the TDOA is estimated correctly, as both of the waveforms are perfectly superimposed and oscillate similarly. On the contrary, the cross-correlation misestimated the TDOA by a number of samples equivalent to one oscillation period (Fig. 10c). Despite both signals oscillating the same way and at the same speed, we see that they are not perfectly superimposed. Therefore, this visual inspection allowed validation of the estimated value obtained with the CRP, while discarding that obtained with the cross-correlation.

For most of the annotated sounds, checking simultaneously all of the transitive relations and the alignment of the waveform after compensation of the TDOAs helped to determine the validity of the estimated TDOAs.

\subsection{Results}

Among the 56 annotated sounds, the CRP-based method succeeded in the estimation of all six TDOAs for 18 of them, which represents a success rate of $32.1 \%$. The classical cross-correlation succeeded in the estimation of all six TDOAs of only 10 annotated sounds, which represents a $17.6 \%$ success rate. This first observation suggests that the proposed TDOA estimator gives better performances than that using the cross-correlation. This apparent low success rate obtained for the estimation of all of the TDOAs of singular annotated sounds can be explained according to the 

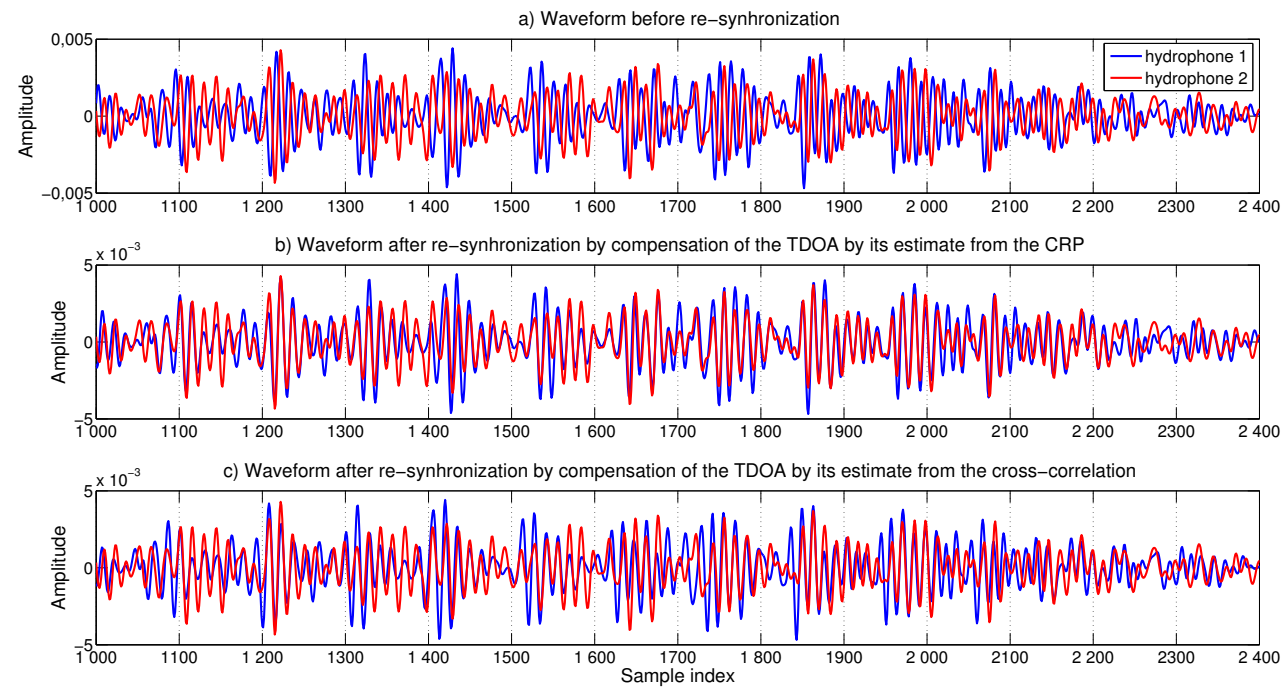

Fig. 10: Examples of waveforms received simultaneously by two distinct hydrophones. The signal from hydrophone 1 is shown in blue, and the signal from hydrophone 2 is shown in red. (a) Before the signals were re-synchronized. (b) After the signals were re-synchronized by compensating for the TDOA by the estimated value obtained with the CRP. (c) After the signals were re-synchronized by compensating for the TDOA by the estimated value obtained with the cross-correlation. We note that for this particular example, the CRP estimated the TDOA well, whereas the cross-correlation failed to estimate the TDOA correctly.

two following reasons: First, the periodic nature of the signal makes it very likely to have mis-estimated the TDOA values. The error is then proportional to the signal period. Secondly, the underwater environment is a highly fluctuating and noisy environment, which heavily affects the form of the acoustic signals received by the hydrophones of the antenna, even when the distance between these hydrophones is very small. Therefore, despite the apparent good SNR of the annotated signals, the waveforms recorded are very different from one hydrophone to another, as shown on Figure 9, which makes the TDOA estimation difficult.

Consequently, 38 of the annotated sounds had at least one TDOA misestimated by the CRP-based estimator, and 46 sounds had at least one TDOA misestimated by the cross-correlation. For these sounds, the results for the transitive relations were studied closely, and visual checking was used to find the misestimated TDOA values.

The overall results of this analysis include all 336 estimated TDOAs from the 56 annotated sounds, which show that $77.6 \%$ of the TDOAs were correctly estimated 
by the CRP, while $70.2 \%$ of the TDOAs were correctly estimated by the crosscorrelation.

Therefore, the proposed CRP method provides a gain of $7.4 \%$ for the number of correctly estimated TDOAs, with respect to the cross-correlation. Thus, these results validate the use of the CRP as a tool to estimate the TDOA of signals received by spatially separated sensors.

\section{Conclusion}

In this article, we proposed a method based on CRPs and on dedicated RQA measures to estimate the TDOA of signals that arrive at spatially separated sensors.

Instead of computing the RQA measures on the whole CRP, we computed them on each diagonal parallel to the main diagonal to deduce the TDOA. We used four existing RQA measures from the literature, and we proposed two new RQA measures. Among the investigated RQA measures, only the proposed RQA measure $S S$, which gives the sum of all similarity values that lead to recurrence points in a given diagonal of the CRP matrix, appeared to be appropriate and reliable to correctly estimate the TDOAs.

The proposed TDOA estimator was tested and validated on simulated and real data. The simulated data helped to confirm the reliability and the effectiveness of the proposed method with controlled data for which the true TDOA to be estimated was known. Also, these simulations let us study and understand the influence of the parameters, such as the embedding dimension $m$, the similarity function $\operatorname{Sim}(.$, .), and the RQA measures, on the performances of the proposed method. In particular, the PCC appeared to be the most appropriate similarity function for our purpose. Moreover, the simulated data were also used to compare the CRP-based TDOA estimator with a cross-correlation based estimator. This comparison showed that for signals with weak amplitude modulations, the cross-correlation was better than the proposed method (from $0 \%$ to $25 \%$ higher, depending on the parameters of the simulations and the SNR), whereas for signals with strong amplitude modulations, the CRP using the PCC gave a higher number of correctly estimated TDOAs than the cross-correlation ( $0 \%$ to $30 \%$ higher depending on the parameters of the simulations and the SNR).

All mis-estimated TDOAs obtained with the proposed method were due to a shift of samples that was proportional to the oscillation period of the signal. The periodicity of the simulated and real signals gave several parallel lines on the CRP, and the distance between these diagonals corresponded exactly to the oscillation period of the signal. Due to the high sensitivity of the proposed method, most of the time we can obtain parts or all of these diagonals, even when the signal is highly modulated or has a poor SNR. However, we then face the problem of picking up the diagonal that corresponds to the true TDOA, with an ambiguity factor related to the oscillation period of the signal. This constitutes the major challenge and the potential downfall faced when using the CRP as a TDOA estimator. 
Also, these results appear to be important in the choice and application of a TDOA estimation method on real data. For signals with weak amplitude modulations, the classical cross-correlation or the CRP with the dot product as a similarity function might be sufficient to achieve good performances. On the contrary, more distorted signals would require the use of CRP with PCC to achieve good performances. In the case of underwater acoustics, these concepts of weak and strong amplitude modulations can be related to three different acoustic phenomena: (1) controlled variations in the amplitude of the sound during its emission by the ani$\mathrm{mal}$; (2) amplitude modulations introduced during the propagation due to the reflections and scattering at the boundaries, the frequency-dependent attenuation in the sea, and the distance between the source and receiver; and (3) the directivity of the sound source that affects the waveform 'viewed' by the different hydrophones.

The proposed method was validated on real acoustic data recorded at sea by a four-hydrophone array. As with the simulated data, we showed that the CRP-based TDOA estimator gave better performances than the cross-correlation, with a global score of $77 \%$ of correct estimates for the CRP, as a gain of $7.4 \%$ over the number of correctly estimated TDOAs with respect to the cross-correlation

Acknowledgements The authors would like to thank the DGA for supporting the postdoctoral scholarship of O. Le Bot, the Lyonnaise des Eaux and the Water Agency of Rhone-MediterraneanCorsica for supporting the project SEAcoustic during which the acoustic data were recorded, the research team STARESO based in Calvi, and Julie Lossent for technical support during the recording of the data in the Bay of Calvi (France).

\section{Appendix}

This appendix deals with the automatic controls performed to determine the validity of TDOA estimated on real acoustic data.

This automatic checking is based on the concept that each annotated signal gives six TDOA values, which are not independent of each other, but which must respect transitive relations. A four-hydrophone array gives four independent transitive relations between all of the TDOA values (Fig. 11). Let $T D O A_{i j}$ be the TDOA between hydrophones $i$ and $j$, with $(i, j) \in\{1,2,3,4\}$ the index of the hydrophones. The four independent transitive relations between the estimated TDOA (expressed as samples) are given by:

$$
\left\{\begin{array}{l}
T D O A_{12}+T D O A_{23}=T D O A_{13} \pm 2 \\
T D O A_{12}+T D O A_{24}=T D O A_{14} \pm 2 \\
T D O A_{13}+T D O A_{34}=T D O A_{14} \pm 2 \\
T D O A_{23}+T D O A_{34}=T D O A_{24} \pm 2
\end{array}\right.
$$

As each TDOA was estimated with 1 -sample precision (equiv. $10^{-4} s$ ), the sum of two TDOAs is estimated with 2 -sample precision (equiv. $2 \cdot 10^{-4} \mathrm{~s}$ ). 


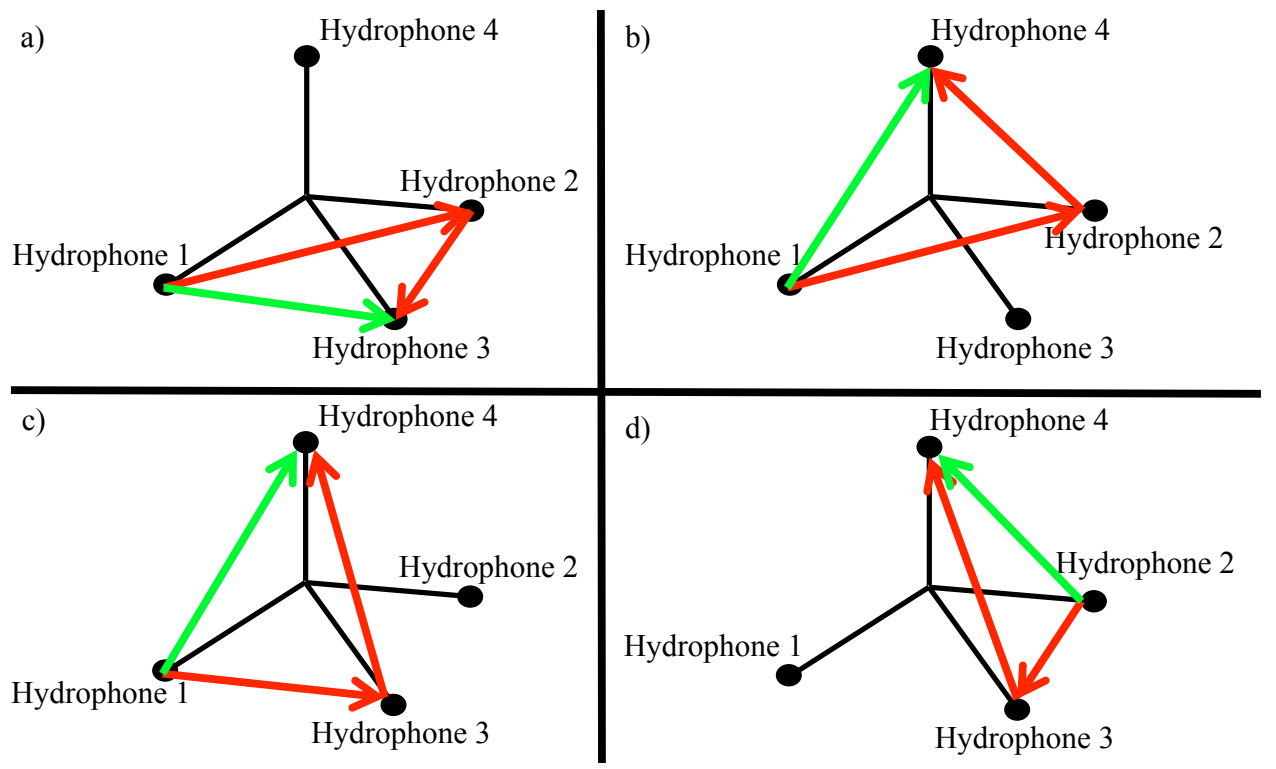

Fig. 11: Four examples of independent transitive relations of the TDOAs given by Eq. (18): a) $T D O A_{12}+T D O A_{23}=T D O A_{13}$; b) $T D O A_{12}+T D O A_{24}=T D O A_{14}$; c) $T D O A_{13}+T D O A_{34}=T D O A_{14} ;$ d) $T D O A_{23}+T D O A_{34}=T D O A_{24}$.

If the four relations given in Equation (18) are met, then we can assume that all of the estimated TDOAs are true. Visual inspection is then performed to confirm the validity of the estimates, and particularly to check whether there are any cumulative errors within the relations; i.e., if two or more TDOAs are misestimated but their sum respects the transitive relations.

If one of the transitive relations from Equation (18) is not met, we check the transitive relations of the third orders, to determine which TDOA is true and which TDOA is misestimated. The TDOA values give three independent transitive thirdorder relations, which are given by:

$$
\left\{\begin{array}{l}
T D O A_{13}+T D O A_{34}-T D O A_{24}=T D O A_{12} \pm 3 \\
T D O A_{12}+T D O A_{23}+T D O A_{34}=T D O A_{14} \pm 3 \\
T D O A_{13}-T D O A_{23}+T D O A_{24}=T D O A_{14} \pm 3
\end{array}\right.
$$

For the relations in Equation (19), the sum of three TDOAs is estimated with 3samples precision. If one of the relations in Equation (19) is verified, then the four associated TDOA values are assumed to be true and a visual inspection is performed to confirm the results. Again the visual inspection aims to prevent cumulative errors.

Finally, for each transitive relation from Equation (19) taken individually, two of the estimated TDOAs are not involved. Either these two estimated TDOAs are false, or only one of them is false. To determine which of these two TDOAs was 
effectively misestimated, we looked back at the transitive relations given in Equation (18) by assuming that the four estimated TDOAs involved in the third-order transitive relations have already been validated.

For example, if the relation $T D O A_{12}+T D O A_{23}+T D O A_{34}=T D O A_{14} \pm 3$ is met, we assume that $T D O A_{12}, T D O A_{23}, T D O A_{34}$ and $T D O A_{14}$ are true. In this transitive relation, we note that $T D O A_{13}$ and $T D O A_{24}$ have not been used. Then, to check whether $T D O A_{13}$ was well estimated, we look to see whether the following relations are also true: $T D O A_{13}=T D O A_{12}+T D O A_{23} \pm 2$ and $T D O A_{13}=$ $T D O A_{14}-T D O A_{34} \pm 2$. If these are both true, it means that $T D O A_{13}$ was correctly estimated. We can do the same for $T D O A_{24}$ by checking whether $T D O A_{24}=$ $T D O A_{14}-T D O A_{12} \pm 2$ and $T D O A_{24}=T D O A_{23}+T D O A_{34} \pm 2$ are satisfied. We repeat this reasoning for the other two transitive third-order relations.

\section{References}

1. B.D. Van Veen, K.M. Buckley: Beamforming: A versatile approach to spatial filtering. IEEE ASSP magazine, 5 (2), 4-24 (1988)

2. R.O. Schmidt: A signal subspace approach to multiple emitter location and spectral estimation", Ph.D. Dissertation, Stanford University, Stanford, CA (1981)

3. R. Roy, T. Kailath: ESPRIT-estimation of signal parameters via rotational invariance techniques. IEEE Transactions on Acoustics, Speech and Signal Processing, 37 (7), 984-995 (1989)

4. K.T. Wong, M.D. Zoltowski: Uni-vector-sensor ESPRIT for multisource azimuth, elevation, and polarization estimation IEEE Transactions on Antennas and Propagation 45 (10), 1467 1474 (1997)

5. P.R. White, T.G. Leighton, D.C. Finfer, C. Powles, O.N. Baumann: Localisation of sperm whales using bottom-mounted sensors. Applied Acoustics, 67 (11-12), 1074-90 (2006)

6. P. Giraudet, H. Glotin: Real-time 3D tracking of whales by echo-robust precise TDOA estimates with a widely-spaced hydrophone array. Applied Acoustics, 67 (11-12), 1106-1117 (2006)

7. Y. Simard, N. Roy: Detection and localization of blue and fin whales from large-aperture autonomous hydrophone arrays: a case study from the St. Lawrence estuary. Canadian Acoustics, 36 (1), 104-110 (2008)

8. L. Houégnigan, S. Zaugg, M. van der Schaar, M. André: Space-time and hybrid algorithms for the passive acoustic localisation of sperm whales and vessels. Applied Acoustics, 71 (11), 1000-1010 (2010)

9. C. Knapp, G.G. Carter: The generalized correlation method for estimation of time delay. IEEE Transactions on Acoustics Speech and Signal Processing, 24 (4), 320-327 (1976)

10. J. Chen, J. Benesty, Y. Huang: Time delay estimation in room acoustic environments: an overview. EURASIP Journal on applied signal processing, 1-19 (2006)

11. , W.C. Knight, R.G. Pridham, S.M. Kay: Digital signal processing for sonar. Proceedings of the IEEE, 69 (11), 1451-1506 (1981)

12. G. Le Touzé, B. Nicolas, J.I. Mars, P. Roux, B. Oudompheng: Double-Capon and double-MUSICAL for arrival separation and observable estimation in an acoustic waveguide.EURASIP Journal on Advances in Signal Processing, 2012 (1), 1-13 (2012)

13. M. Alam, J.H. McClellan, W.R.Scott Jr: Spectrum analysis of seismic surface waves and its applications in seismic landmine detection. J. Acoust. Soc. Am., 121 (3), 1499-1509 (2007)

14. H. Krim, M. Viberg: Two decades of array signal processing research: the parametric approach. IEEE Signal Processing Magazine, 13 (4), 67-94 (1996) 
15. J.P. Zbilut, A. Giuliani, C.L. Webber: Detecting deterministic signals in exceptionally noisy environments using cross-recurrence quantification. Physics Letters A, 246 (1-2), 122-128 (1998)

16. N. Marwan, M. Thiel, N.K. Nowaczyk: Cross Recurrence Plot Based Synchronization of Time Series. Nonlinear Processes in Geophysics, 9 (3-4), 325-331 (2002)

17. N. Marwan, J.Kurths: Nonlinear analysis of bivariate data with cross recurrence plots. Physics Letters A, 302 (5-6), 299-307 (2002)

18. N. Marwan, M.C. Romano, M. Thiel, J. Kurths: Recurrence plots for the analysis of complex systems. Physics Reports, 438 (5), 237-329 (2007)

19. N. Marwan, M.H. Trauth, M. Vuille, Mathias, J Kurths: Comparing modern and Pleistocene ENSO-like influences in NW Argentina using nonlinear time series analysis methods. Climate Dynamics, 21 (3-4), 317-326 (2003)

20. J. P. Eckmann, S.O. Kamphorst, D. Ruelle: A new graphical tool for measuring the time constancy of dynamical systems is presented and illustrated with typical examples. Europhysics Letters, 4 (91), 973-977 (1987).

21. N. H. Packard, J.P. Crutchfield, J.D. Farmer, R.S. Shaw: Geometry from a time series. Physical Review Letters, 45 (9), 712-716 (1980)

22. F. Taken: Detecting strange attractors in turbulence. Dynamical systems and turbulence, Lecture Notes in Mathematics, 898, 366-381 (1981).

23. O. Le Bot, C. Gervaise, J.I. Mars: Similarity matrix analysis and divergence measures for statistical detection of unknown deterministic signals hidden in additive noise. Physics Letters A, 379 (40-41), 2597-2609 (2015)

24. J.P. Zbilut, C.L. Webber: Embeddings and delays as derived from quantification of recurrence plots. Physics letters A, 171 (3), 199-203 (1992)

25. C.L. Webber, J.P. Zbilut: Dynamical assessment of physiological systems and states using recurrence plot strategies. Journal of applied physiology, 76 (2), 965-973 (1994)

26. N. Marwan, N. Wessel, U. Meyerfeldt, A. Schirdewan, J. Kurths: Recurrence-plot-based measures of complexity and their application to heart-rate-variability data. Physical review E, 66 (2), $026702(2002)$

27. F.M. Birleanu, C. Ioana, C. Gervaise, A. Serbanescu, J. Chanussot, Jocelyn: Caractérisation des signaux transitoires par l'analyse des récurrences de phase. XXIIIème colloque GRETSI (2011)

28. O. Le Bot: Détection, localisation, caractérisation de transitoires acoustiques sous-marins. Thse de l'Université de Grenoble (2014) 